\title{
MUJERES Y TRABAJOS EN EL SIGLO XXI: UNA APROXIMACIÓN DESDE DATOS SECUNDARIOS
}

\author{
Women and works in the 2 I st century: an approach from statistical data
}

Gabriela Vergara'

\begin{abstract}
Resumen
En este artículo contextualizamos el mundo del trabajo a partir de la descripción de variables sociodemográficas provenientes de datos secundarios disponibles para una ciudad del interior de Argentina. Dicho propósito se basa en el interés por conectar procesos estructurales que inciden en las mujeres trabajadoras y, en su doble presencia. Para ello, en el primer apartado explicitamos una red conceptual desde la estructuración social, la producción/reproducción social y, la condición corporal/sensible de la agencia social. A continuación, hacemos mención a tres procesos de estructuración social que vinculan a las mujeres y el trabajo. En un tercer momento, describimos e interpretamos datos secundarios en torno a tres ejes: población, hogares y trabajo. En las consideraciones finales identificamos tres procesos convergentes: a) el contraste en términos demográficos y de formalidad entre un grupo etario de adultos mayores por un lado, y jóvenes adultos con ocupaciones informales, incluido el cuentapropismo, por otro; b) la presencia de empleo público femenino y la feminización de la docencia como uno de los lugares en los que persisten estabilidad laboral y empleo formal y, c) hogares sin vivienda propia, con jefatura de hogar femenina en aumento y menor cantidad de hijos. La interrelación de estos fenómenos podría indicar un paulatino deterioro del empleo estable y la persistencia de ocupaciones con menores niveles de estabilidad que dan cuenta de una desarticulación de la sociedad salarial con una fuerte presencia de las mujeres en el mundo del trabajo y en los hogares.
\end{abstract}

Palabras clave: Mujeres; Trabajos; Estructuración social.

\begin{abstract}
In this article we contextualize the world of work based on the description of sociodemographic variables from secondary data available for a city in the interior of Argentina. This purpose is based on the interest in connecting structural processes that affect working women and their double presence. To do this, in the first section we explain a conceptual network from social structuring, social production / reproduction and, the bodily / sensitive condition of the social agency. Next, we mention three social structuring processes that link women and work. In a third moment, we describe and interpret secondary data around three axes: population, households and work. In the final considerations we identify three convergent processes: a) the contrast in demographic and formality terms between an age group of older adults on the one hand, and

1 Doctora en Ciencias Sociales (UBA), investigadora adjunta de CONICET con lugar de trabajo en la Universidad Nacional de Villa Maria (UNVM), investigadora del Centro de Investigaciones y Estudios Sociológicos (CIES). E- mail: vergara.gabriela@outlook.com.
\end{abstract}


young adults with informal occupations, including self-employment; b) the presence of female public employment and the feminization of teaching as one of the places where labor stability and formal employment persist and, c) homes without their own homes, with female heads of households increasing and fewer children. The interrelationship of these phenomena could indicate a gradual deterioration of stable employment and the persistence of occupations with lower levels of stability that account for a disarticulation of the salary society with a strong presence of women in the world of work and homes.

Keywords: Women; Works; Social structuration.

\section{Introducción}

Las maneras en que se distribuyen los cuerpos con sus distancias y capacidades de acción, las formas que adquieren las relaciones sociales y las sensibilidades tienen en las lógicas de la producción bajo ganancia por un lado y, en la de la vida por otro, dos momentos de análisis particulares en sí mismos y, otro más, en sus intercambios. Si este análisis se espacializa en centros urbanos intermedios, de menos de 100.000 habitantes que han pasado por etapas de industrialización, desindustrialización y reprimarización de las exportaciones, en otros procesos, podemos abrir un conjunto de interrogantes respecto de los procesos de estructuración social.

En otro lugar (VERGARA, 2018a) identificamos a partir de las experiencias de trabajadores en San Francisco (Córdoba, Argentina), un conjunto de etapas que permiten comprender desde lo cotidiano, las metamorfosis del mundo del trabajo y sus sensibilidades. La pujante vida industrial y comercial de la ciudad organizada desde el modelo de Industrialización por Sustitución de Importaciones (ISI) se frenó de manera abrupta a finales de los '80. El paso de las horas extras a las suspensiones y la venta de lapiceras chinas en la calle fue un indicio pequeño y cotidiano de la apertura de importaciones y fin del bien-estar. En la siguiente década, la ciudad fue la "ausencia de", el cierre o las quiebra, junto con changas, informalidad y la importación de bienes de consumo. Las continuidades se dieron en virtud de un proceso que se extendió desde los '80 hasta fines de la década siguiente. En un tercer momento, en los primeros años del 2000 observamos una desconexión importante operada en los esquemas de percepción de las personas entrevistadas que oscila entre la so portabilidad y la 
naturalización: antes había mucho trabajo y ahora también, pero el tipo de trabajo no es el mismo. Las condiciones de contratación, las calificaciones para los trabajadores donde hay trabajo son diferentes. Y si tener trabajo cambió el "ánimo", se debe a que las sensibilidades se reajustaron a estas nuevas condiciones de expropiación de energías que se han intensificado para la claseque-vive-del-trabajo. Es decir que la actividad económica, a decir de los agentes se fue recuperando, encaminando y creciendo, pero, de la mano de trabajo en negro, aportes menores a las horas trabajadas e informalidad, en medio de una lógica de desechar la mano de obra -como cualquier otro objeto que cuando no sirve, se desecha. Si antes todos estaban bien porque habia mucho trabajo, ahora el bienestar empieza a pasar por poder seguir consumiendo, aunque sea objetos cada vez más baratos.

En este marco, también hemos analizado percepciones de trabajadoras configuradas en el cruce entre sus trayectorias de clase y las metamorfosis del mundo del trabajo, donde se observan diversidad de formas de expropiación de energías en la polivalencia de tareas y la informalidad de ocupaciones. Esto opera en medio detenciones entre (lo que aparece) como opciones en la estabilidad del empleo público y lo polifacético del sector servicios; entre la doble jornada para las mujeres y el mantenimiento del hogar con un varón proveedor (VERGARA, 2018b).

$\mathrm{Si}$ el mundo del trabajo presenta tensiones, metamorfosis $\mathrm{y}$ heterogeneidades que en el caso de las mujeres se intensifican, nos preguntarnos qué sucede en las interrelaciones con el mundo de la reproducción de la vida en general y de la fuerza de trabajo en particular.

El aludido análisis se inscribe en un proyecto de investigación titulado "Prácticas y sensibilidades de mujeres adultas no profesionales de San Francisco en sus trabajos productivos y reproductivos en la actualidad" (20182019)2. El objetivo general de dicho proyecto es comprender las formas de

\footnotetext{
2 Aprobado por el Instituto de Investigación de la Universidad Nacional de Villa María (UNVM), dirigido por la Dra. Gabriela Vergara, para el período 2018-2019. Dicha indagación forma parte del plan de trabajo de CONICET "Estructuración social, tramas corporales y sensibilidades en mujeres trabajadoras no profesionales de Villa María, San Francisco y Rafaela (2018-2019)".
} 
estructuración social del mundo del trabajo, la vida cotidiana y sus interrelaciones a partir de las prácticas y sensibilidades de mujeres trabajadoras adultas de San Francisco, en la actualidad.

En este artículo nos centramos en el primer objetivo específico del aludido proyecto, que busca contextualizar el mundo del trabajo en la ciudad considerando la variable de género. Es decir, el interés está puesto en describir variables sociodemográficas a partir de los datos disponibles para la ciudad, en el intento por conectar procesos de carácter estructural que inciden en las mujeres trabajadoras, como así también en las características de su doble presencia. Para ello desarrollaremos la siguiente estrategia argumentativa: en el primer apartado explicitamos una red conceptual desde la estructuración social, la producción y reproducción social y, la condición corporal/sensible de la agencia social. A continuación, hacemos mención a tres procesos de estructuración social que vinculan a las mujeres y el trabajo. En un tercer momento, describimos e interpretamos datos secundarios en torno a tres ejes: población, hogares-viviendas y trabajo. En las consideraciones finales presentamos algunos interrogantes en relación con las tensiones entre la producción y la reproducción social.

\section{Estructuración y condición corpóreo-sensible de la agencia social}

En el intento por articular perspectivas teóricas sociológicas y análisis de género (que buscan en las perspectivas clásicas de la economía una crítica a las teorias neoclásicas que solo ven a la fuerza de trabajo como costo de producción) podemos considerar al menos, dos niveles.

Por un lado, el concepto de estructuración social, sensu Giddens, como producción/reproducción de prácticas e instituciones. Esta idea permite incorporar una dimensión de una reproducción social de la desigualdad (en el sentido de Bourdieu) en términos de raza, género, edad, clase social. Una actividad de la sociedad que no solo reproduce o continuación las mismas estructuras, sino que éstas se van modificando, sobre todo por la reflexividad del agente diestro, o porque sus esquemas de percepción pueden cambiar ante nuevas situaciones - ajustes del habitus. 
Dicha estructuración social en su forma histórica capitalista (neocolonial, dependiente, extractivista) se basa en la producción de bienes y servicios para la obtención de ganancia y acumulación. Para esto se vale de la reproducción de la fuerza de trabajo humana (subsumida como una mercancía) por un lado. Por otro, precisa de la reproducción (biológica, cotidiana y social de la fuerza de trabajo que tradicionalmente ha acontecido en los hogaresfamilias y tuvo como pivote central al trabajo - no remunerado - de las mujeres (abuelas, tías, madres, hermanas, amigas, vecinas) para sostener la lógica de la vida. Esto se mantuvo y se mantiene hasta la actualidad pese al incremento de la cantidad de mujeres en la fuerza de trabajo, configurando una doble presencia o jornada.

En este marco se entabla una relación compleja entre la reproducción y la producción dado que "el proceso de acumulación utiliza las energías humanas como mercancías y la tarea del trabajo doméstico es reproducir esas energias como parte integrante de las personas, tarea que sin embargo debe desarrollarse dentro de los límites de su reproducción como mercancías" (PICCHIO, 1994, p. 455).

Las tensiones que se generan entre estas dos lógicas opuestas en los pliegues de la vida cotidiana y doméstica pueden ser comprendidas desde una Sociología de los cuerpos y las emociones. Desde esta perspectiva asumimos que los agentes sociales que participan y hacen a la producción/reproducción social como del sistema capitalista, pueden ser definidos desde su condición corporal y, que los cuerpos en el trabajo, en el capitalismo del siglo XXI siguen asistiendo a una expropiación de energías. Cuando decimos cuerpo, lo entendemos como una trama de dimensiones sociales, subjetivas y sociobiológicas, influidas por sus biografias, por su posición y condición de clase que llamamos tramas corporales. Esta expropiación ubica al cuerpo como lugar del conflicto y del orden, y por tanto podemos identificar cómo las sensibilidades operan, actúan en la naturalización de las desigualdades para restablecer el orden y generan cierta so portabilidad social. 
En el mundo del trabajo hay una heterogeneidad de formas desde donde se produce la expropiación de energías que puede ser pensada en términos de una clase-que-vive-del-trabajo (ANTUNES, 2005) o, que sobre-vive con ese trabajo. La expropiación y la so portabilidad nos permiten considerar el lugar de las sensibilidades que se configuran permitiendo la segunda. En trabajos previos hemos identificado las sensibilidades de los desechables (VERGARA, 2012), de los sobrevivientes y de los surfientes (VERGARA, 2018c), en distintos segmentos del mundo del trabajo, más o menos precarios. Dichas categorias operan como hipótesis para comprender la naturalización de una expropiación de energías cada vez más intensa.

En el siguiente apartado focalizamos algunos aspectos que afectan particularmente a las mujeres.

\section{Mujeres y trabajos}

La feminización del mercado de trabajo puede ser entendida como el proceso por el cual se verifica un aumento progresivo de las mujeres en trabajos remunerados fuera de sus hogares, en economías modernas. Determinadas series estadísticas a nivel internacional mostraban que, a fines del siglo XIX, en sociedades tradicionales basadas en el autoabastecimiento las mujeres tenían una elevada participación en la producción de bienes y servicios, pues la familia era la unidad donde se realizaban concomitantemente las actividades domésticas y económicas (RECHINI DE LATTES Y WAINERMAN, 1977). En Argentina comenzó a manifestarse a partir de 1970 con una marcada tendencia al aumento.

En este marco, los mercados de trabajo persistieron en su división del trabajo por géneros distinguiendo en el mismo ámbito público - otrora vedado para las amas de casa- entre ramas, sectores, y puestos. Así, por ejemplo, los ámbitos destinados al cuidado, a la enseñanza de los más pequeños fueron predominantemente femeninos. La docencia, por ejemplo, se asoció una imagen maternal, donde la escuela era el segundo hogar, y, por ende, la maestra, la 'segunda mamá' (WAINERMAN Y NAVARRO, 1979). Estos procesos se conjugaron para que a fines del siglo XX las familias cuenten con dos fuentes de ingresos o dos proveedores de manera estable, lo que se conoce como el dual 
earner model. En América Latina, la participación laboral del cónyuge -mujerpasó del 37\% en 1990 a 47,6\% en el 2002, haciendo que, dentro de las familias biparentales en la mitad de los casos, el ama de casa se constituya en un aporte permanente del ingreso familiar. En Argentina para el mismo período, en familias biparentales urbanas el incremento fue de 35,2\% al 43,2\% (SUNKEL, 2006). Esto implica la emergencia de un nuevo patrón de comportamiento integrado por dos proveedores y una cuidadora (MARTÍN PALOMO, 2008).

La persistencia del monopolio del cuidado de los otros en manos de las mujeres, lleva a identificar como tercer efecto de la feminización laboral en el cruce con la división de trabajo, la constitución de la doble presencia o doble jornada, puesto que los varones no presentan alteraciones en las escasas horas de trabajo doméstico que realizan, aunque aumente el número de hijos o la mujer trabaje, pues en este último caso ella debe asumir "la doble jornada y el doble trabajo, desplazándose continuamente de un espacio a otro, solapando e intensificando sus tiempos de trabajo. Tiempos que vienen determinados, por un lado, por las exigencias de la producción mercantil y, por otro, por los requerimientos naturales de la vida humana" (CARRASCO, 2003, p. 37). En esta línea no es menor que, considerando los censos del período 1991-2010 se registró para Argentina, un aumento de mujeres jefas de hogar, en todos los tipos de hogares, incluso en hogares nucleares con hijos (BINSTOCK, 2013).

En términos de procesos de estructuración social observados en América Latina, en Argentina y, en la ciudad bajo estudio, destacamos tres fenómenos interrelacionados. En primer lugar, nos referimos a la feminización del trabajo, en tanto aumento constante en las últimas cuatro décadas de participación de mujeres dentro de la población ocupada. Una parte importante de éste, ha sido absorbido por el capital en trabajos part-time, desregulados, informales, con salarios y derechos desiguales con respecto el trabajo masculino.

Además, esto se da en un contexto de segregaciones horizontales y verticales que se entrelaza con el dilema de la necesidad o la libertad y, se inscribe en una trama de vectores de posiciones y condiciones de clase. En este 
marco, la creación de puestos de trabajo para mujeres en un contexto de globalización generó empleos precarios y vulnerables, reforzando una división del trabajo y desigualdades sociales en términos de géneros, razas y clases sociales. Dentro de la división del trabajo, se identifican como nuevas configuraciones la bipolarización del empleo femenino y nuevos modelos de conciliación entre la vida familiar y profesional (HIRATA, 2010). En España por ejemplo se ha identificado la persistencia de discontinuidades en las trayectorias de las mujeres y mayor precariedad en el mundo del trabajo, lo cual es asumido como resultante de mutaciones del patriarcado (ALCAÑIZ MOSCARDÓ, 2017), mientras que en Brasil se ha registrado una polarización en la inserción de las mujeres. De un lado, un grupo con nivel educativo al toque, en Brasil para 2010 registraba una tasa de ocupación elevada y con una modalidad de jornada completa, igualándose en este segmento educativo con los varones. Pero, por fuera de esta variable, era mayor el porcentaje de mujeres respecto de los varones que trabajan de manera informal. Pese a que un reducido grupo de mujeres logra satisfactorias inserciones, la amplia mayoría continua en puestos menos valorizados (tiempo parcial, ocupaciones informales y vulnerables y, con salarios bajos) (GUEDES, 2015).

Por otra parte, consideramos que las políticas sociales generan formas de sociabilidad y a la vez, reducen los conflictos entre clases sociales. En el Sur Global, la feminización de la pobreza intensificó programas que pretenden reducir dicho fenómeno a través de la promoción del trabajo para las mujeres. Dichas actividades adquieren, en la mayoría de los casos, las características de ser informales, precarias, de baja calificación, o en el marco del cuentapropismo de oficio. En otro lugar (VERGARA, COLOMBO, MANAVELLA Y PEÑARRIETA, 2018) hemos identificado un conjunto de políticas sociales que en Córdoba se formularon explícitamente con este objetivo. En cuanto a las políticas impulsadas por el Banco Mundial de emprendedorismo y microcréditos, éstas han puesto a las mujeres en el centro de atención, bajo el supuesto de que son agentes activos del desarrollo. Frente a ello, se ha cuestionado que el emprendedorismo de las mujeres encubre en realidad, un modelo neoliberal competitivo, privatizador y de desarrollo basado en el 
mercado (BOERI, 2018). Por otra parte, la feminización de las políticas sociales en Argentina encuentra una de sus primeras manifestaciones en la década del $80^{\circ}$, de la mano del comunitarismo y la descentralización. Tras un período de focalización de las políticas sociales con muy pocos resultados positivos, en los inicios del presente siglo se pasó a modelos de masividad. Pero a comienzos de este siglo XXI, impulsados por organizamos internacionales, se observan programas destinados a familias o hijas/hijos cuyos requisitos de acceso reafirman "la distribución sexual del trabajo al interior de la familia que carga a la mujer con la "doble tarea" (DE SENA, 2014, p. 102). A modo de ejemplo, el plan Jefas y Jefes de hogares desocupados iniciado en 2002 en Argentina, mostró desde esa fecha hasta 2006 un crecimiento del $50 \%$ de beneficiarias mujeres, pasando de ser el 40\% en 2002, al 63\% en 2006 (DE SENA, 2014).

En tercer lugar, asistimos a la persistencia de las mujeres en el trabajo no remunerado o de reproducción. En el marco de los dos procesos anteriores (feminización del trabajo y de las políticas sociales - vía feminización de la pobreza), se mantiene como tendencia que las mujeres con trabajo asalariado tengan, a su vez, una mayor cantidad de horas de trabajo doméstico, comparando con sus cónyuges en similar condición. Esto ha sido registrado desde hace más de 4 décadas en Europa y Estados Unidos. Asípues, según datos de Italia para 1985, el promedio de horas semanales dedicadas al trabajo doméstico, era de 5 para varones frente a 36 horas en las mujeres. Los hombres en pareja registraron la mitad del trabajo doméstico comparados con quienes viven solos. El nacimiento de un hijo aumentaba más el trabajo doméstico en horas, si la mujer no tenía trabajo remunerado, pero los hijos no son la única causa de aumento de trabajo remunerado. A mayor número de integrantes disminuye el de hombres y aumenta el de mujeres. Los datos indican comportamientos tan dispares que parecen mostrar que viven en dos tipos de familias diferentes. La feminización del trabajo, la reducción del número de hijos y la introducción de electrodomésticos fueron identificados como factores de una tendencia a la reducción del trabajo doméstico y en el caso de los hombres un leve incremento. En la crítica a la teoría neoclásica de 
Becker (racionalización de la acción para maximizar utilidad marginal), se destaca que la mercancía fuerza de trabajo tiene una especificidad social y política para su reproducción. En el proceso social de la reproducción de la fuerza de trabajo intervienen factores tales como el Estado, costumbres, gustos (PICCHIO, 1994).

Las dinámicas de estos procesos interrelacionados llevan a preguntarnos por el estado de otras variables, desde las tasas de envejecimiento de la población, pasando por el número de hijos por hogar. En el apartado que sigue, presentamos una descripción de un conjunto de variables que nos permitirán contextualizar complementar lo expuesto hasta aquí.

\section{Análisis de procesos de estructuración desde datos secundarios}

En este apartado presentamos un análisis descriptivo de diferentes variables que nos permiten describir de modo general algunos procesos que caracterizan al mundo del trabajo y al mundo de las familias-hogares en la ciudad de San Francisco (Córdoba, Argentina). Dicha contextualización parte de considerar a la ciudad, desde un doble sentido. Por un lado, en su particularidad, desde el interés por conocer sus características específicas dado su perfil productivo, su ubicación geográfica, su historia, entre otras. Por otro, desde su generalidad, pues en un sentido metonímico, como ciudad neocolonial sensu Scribano, da cuenta de procesos de estructuración social de vasto alcance.

Dada la disponibilidad de información para el área de interés, analizamos las siguientes fuentes de datos secundarios: Censo de la Provincia de Córdoba 2008; Censo Nacional de Población y Vivienda 2010 con datos desagregados para San Francisco; Encuesta Anual de Hogares Urbanos de Córdoba; Relevamiento del mercado laboral para el conglomerado San Francisco-Frontera-Josefina 2009. A continuación, repasamos características de la provincia, para luego centrarnos en la ciudad/aglomerado aludida.

\subsection{Córdoba}


Según la Encuesta Anual de Hogares Urbanos en Córdoba, la tasa de empleo (porcentaje entre población ocupada y población total) se ubicó en el $52 \%$ para 2010 y en el 54,3\% en 2014. En particular, la tasa de empleo de mujeres en igual período osciló entre el 41\% y el 43\%, aunque en el rango etario de 30-64 años esta tasa fue más elevada, ubicándose entre el 56\% y el $63 \%$. Esto último da cuenta de la importante presencia de mujeres adultas en el mercado de trabajo, en un período de la vida biológico-social en el cual muchas personas integran hogares con parejas e hijos.

Si consideramos el tipo de relación laboral, advertimos que disminuyó el trabajo asalariado en 4 puntos porcentuales (de $73 \%$ en 2010 a 69\% en 2014) y aumentó el cuentapropismo un $6 \%$ (de 20\% a 26\%, respectivamente). Las asalariadas sin descuento jubilatorio representaron el $38,4 \%$ al comienzo del cuatrienio y el $46,5 \%$ al final del mismo, siguiendo la misma tendencia de los varones. En cuanto al sector, más de la tercera parte de las mujeres se desempeñó en el ámbito privado cuya participación tuvo un leve incremento dado que pasó de 79,8\% en 2010, a 83,1\%, 4 años después. Dentro del servicio doméstico, ocupación principalmente femenina, el $76,6 \%$ de las mujeres no estaba registrada en 2010, en tanto que en 2014 el no registro ascendió levemente para ubicarse en 77,6\% (DIRECCIÓN GENERAL DE ESTADÍSTICAS Y CENSOS, S/D).

En líneas generales podemos decir que más mujeres adultas se incorporaron al mercado laboral encontrando ocupaciones en relación de dependencia sin aportes jubilatorios en el ámbito privado o bien, insertándose como cuentapropistas. Como contrapartida, en el periodo antes mencionado el incremento de desempleo se registró entre los deciles más bajos de ingresos, mujeres jóvenes y, entre quienes tenían una ocupación anterior, unos de cada cuatro provenían del sector de la construcción.

Otra fuente de datos secundarios que complementa la descripción del mundo del trabajo precedente, es la Encuesta sobre Trabajo No Remunerado y Uso del Tiempo realizada en 2013 a nivel nacional, cuyos datos para Córdoba, indicaron que las mujeres dedicaron6 horas promedio para trabajo doméstico 
no remunerado y los varones 3,3 horas $^{3}$. Considerando la presencia de al menos 1 hijo menor de 6 años, la tasa de participación de las mujeres en el trabajo no remunerado fue del 92,5\%, mientras que la tasa de participación de los varones fue del 60\%. Teniendo en cuenta la condición de ocupado, el 87,5\% de las mujeres realiza trabajo no remunerado, frente a un 52,8\% de los varones (INDEC, 2014). Esto indica que la doble presencia de las mujeres se inscribió en el período analizado en Córdoba en un contexto laboral atravesado por informalidad, cuentapropismo y desempleo.

\subsection{San Francisco}

En este apartado describimos desde tres ejes interrelacionados, datos relevantes en cuanto a población, hogares-viviendas y trabajo. A partir de las variables descriptas buscamos caracterizar algunos procesos del mundo del trabajo y también de las condiciones de vida de la población, tales como salud, educación, a fin de realizar una primera aproximación a las relaciones entre producción de bienes/servicios y, reproducción de fuerza de trabajo.

\subsection{Población}

Por su ubicación, San Francisco es el principal centro urbano de un aglomerado interprovincial conformado junto con la ciudad de Frontera (10.723 habitantes) y el barrio Acapulco y Veracruz (2.142 personas) de la localidad de Josefina, ambas pertenecientes a la provincia de Santa Fe. Dicho aglomerado presenta características socioeconómicas y productivas heterogéneas (FRAIRE, MAGNANO, COLOMBO Y PEÑARRIETA, 2018), pese a lo cual se traman intercambios de diversa índole más allá de los límites interprovinciales.

Según el Censo 2010, San Francisco fue la cuarta ciudad del interior provincial en cuanto a población se refiere, con 62.211 habitantes, posicionada detrás de Río IV, Villa María y Carlos Paz. En ese periodo se registró un coeficiente de 19,4 que comparado con el provincial de 15,2 indicó una mayor vejez demográfica. Esta menor cantidad de nacimientos en el largo plazo a nivel

\footnotetext{
3 Estudios recientes en Rio de Janeiro indican que se mantiene elevada la imagen de que la familia es la responsable del cuidado de niños y adultos mayores. Sin embargo, se identificaron diferencias en cuanto a género y nivel educativo. Varones con baja o nula educación manifiestan respuestas concordantes al modelo tradicional de varón proveedor y mujer cuidadora, mientras que el mismo segmento educativo en mujeres indican por ejemplo que el Estado debería brindar más oportunidades para el cuidado de niños (GUEDES, 2016).
} 
Mujeres y trabajos en el siglo XXI: una aproximación desde datos secundarios| Gabriela Vergara

provincial, como parte de la transición demográfica indica modificaciones en la conformación de los hogares, como así también en el tipo de cuidados a realizar. La Imagen 1 presenta a la izquierda la pirámide población para toda la provincia de Córdoba en 2010, mientras que a la derecha se ubica la correspondiente a la ciudad de San Francisco.

Imagen 1. Comparación de pirámides de población de Córdoba y San Francisco (2010)
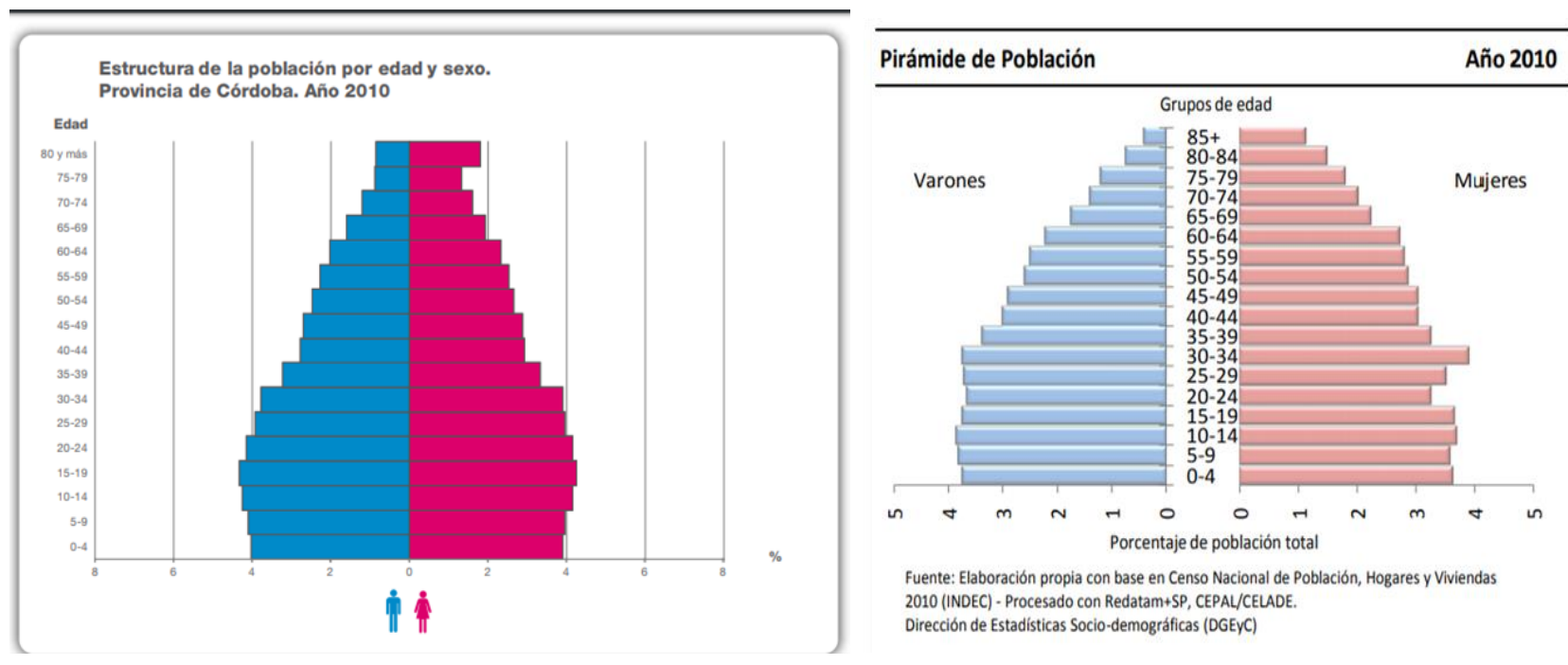

Fuente: Censo de población, 2010. Dirección de Estadísticas y Censos de Córdoba4.

La distribución de la población por edad y sexo muestra una pirámide de envejecimiento con una estructura similar a la provincial 5 . El índice de envejecimiento ${ }^{6}$ es de 86,2 , y considerando solo a las mujeres el índice se eleva a 104,9\%. El índice de masculinidad registra sus valores más bajos en los

\footnotetext{
4 Pirámide de población de Córdoba tomada https://www.indec.gov.ar/comunidadeducativa/censo2010.pdf; pirámide de población de San Francisco, tomada de: https://datosestadistica.cba.gov.ar/dataset/eaab30e4-d400-465d-bf53e9094558f3d2/resource/515c872f-ab6a-480f-8d23-c39a7ba20186/download/desd_cn2010_san_justo_-

san-francisco_cabecera.pdf

5 Argentina se ubica dentro del grupo de países (junto con Uruguay, Chile, Cuba, Barbados, Bahamas, Guadalupe, Jamaica, Martinica y Puerto Rico) con una transición demográfica avanzada, según la tipología del CELADE. En este grupo los países presentan natalidad y mortalidad moderada a baja, por lo cual el crecimiento es bajo. Estimaciones para el quinquenio 2025-2030 indican que para Argentina el crecimiento será del 6.9\%. (CEPAL, https://repositorio.cepal.org/bitstream/handle/11362/39228/1/S1500643_mu.pdf Proyecciones de población. Observatorio Demográfico. 2014.

6 Se calcula como el cociente entre la población de 60 años y más sobre población de 0-14 años, multiplicado por 100 .
} 
grupos etarios de 75-79 y, 80 y más. Esto incide directa e indirectamente con la economía de cuidados y/o con el trabajo de cuidados de los adultos y de las mujeres, en la estructura de los hogares (sea porque demandan cuidados que deben ser resueltos por los hogares, principalmente las mujeres, o bien por servicios de cuidado del sector privado. Por otra parte, genera problemas al sistema previsional en un contexto de empleo registrado asalariado en constante retroceso) como así también plantea desafios para la planificación del equipamiento urbano, es decir la dotación de escuelas, hospitales, parques, teatros, museos, entre otros (NARVÁEZ MONTOYA, 2012).

La población mayor a 65 años registró un 94,6\% de percepción de jubilación y/o pensión, lo cual es considerado como un factor importante que reduce las posibilidades de ser pobre. De hecho, en América Latina se verifica que la presencia de pobreza por ingresos en hogares donde hay adultos mayores es menor que en los hogares donde no los hay (PAZ, 2011).

En relación con lo anterior, el acceso a la cobertura de salud fue mayor en la población adulta bajo sistema de jubilación, donde había más mujeres ancianas vivas. La salud y el sistema previsional fueron parte de una estructura de estado benefactor que paulatinamente se va reduciendo y que presenta en estos segmentos de la sociedad, algunos de sus vestigios. Esto se ve reforzado por el hecho de que el rango con menor cobertura eran los adultos jóvenes (grupo etario en el que hay muchas mujeres, y con empleo sin registrar). En el caso de los niños, 3 de cada 10 no tenían cobertura ${ }^{7}$. Estos datos indican en su reverso, ciertos aspectos del mundo del trabajo en términos de mayor informalidad.

Según el Censo Provincial de Córdoba de 2008, el máximo nivel educativo alcanzado para la población mayor a 18 años en San Francisco, era de hasta secundario incompleto (sin diferencias significativas por género) para1

\footnotetext{
7 Pese a la descentralización de los servicios de salud llevada a cabo en la década del 90, los sectores de la población que no cuentan con cobertura de salud propia pueden acceder a las prestaciones de los hospitales públicos, los cuales han asistido a importantes modificaciones en su funcionamiento, sea por la forma en que deben generar ingresos económicos, como por la heterogeneidad de formas de contratación y empleo de quienes trabajan en ellos (ASE, 2006). El Hospital provincial José Bernardo Iturraspe que posee la ciudad, está definido como de alta complejidad, abarcando atención cardiovascular, de neonatología, neurológica/neuroquirúrgica, de salud mental y geriátrico, con 245 camas (MINISTERIO DE SALUD, 2011).
} 
Mujeres y trabajos en el siglo XXI: una aproximación desde datos secundarios| Gabriela Vergara

de cada 2 personas, mientras que el 25\% de los varones y el $22 \%$ de las mujeres tenía secundario completo. Comparando los niveles educativos por separado observamos una leve diferencia a favor de los varones dado que, mientras había más varones con secundario completo, se registraron más mujeres con primario completo. Si consideramos los niveles terciarios/superior y universitarios completos, encontramos al $11 \%$ de los varones y al $17 \%$ de las mujeres. Al desagregarlos notamos una segunda diferencia a favor de los varones, puesto que el mayor contraste se da en la feminización del nivel terciario, donde el $11 \%$ de las mujeres, había completado el nivel terciario/superior completo, frente al $4,5 \%$ de los varones para el mismo nivel. Por su parte, hay una diferencia de 1 punto en el nivel universitario completo a favor de los varones y de 2 puntos en el universitario incompleto. En líneas generales, había una feminización en niveles inferiores de educación (primario completo) mientras que en el nivel universitario habia mayor representación masculina. La presencia de profesorados en la ciudad y la persistencia de la feminización de la docencia podrian explicar esta tendencia ${ }^{8}$.

En síntesis, tras los datos expuestos precedentemente identificamos en las tres variables lo siguiente: tendencia a envejecimiento con mayor presencia de mujeres, acceso a la salud en grupos etarios mayores fenómeno que no se replica en jóvenes, y niveles educativos inferiores al secundario completo para la mitad de la población al tiempo que una tendencia a la feminización del nivel terciario. A continuación, presentamos variables que indican características de hogares y viviendas.

\subsubsection{Hogares y viviendas}

En función de los datos disponibles, según el Censo Nacional 2010, en San Francisco una de tres mujeres era jefa de hogar. Esta proporción era levemente inferior a los registros provinciales, tal como observamos en el siguiente gráfico:

$8 \mathrm{El}$ ámbito de la educación preescolar sigue siendo predominantemente femenino, al punto de que los varones que participan en el mismo pueden ser vistos como extraños o peligrosos, excepto que desempeñen tareas de representación sindical, asesorías pedagógicas o dirección de escuelas, es decir vinculados con la fuerza y el poder (GARCÍA VILLANUEVA ET AL., 2015). 
Gráfico I. Jefatura de hogar según sexo

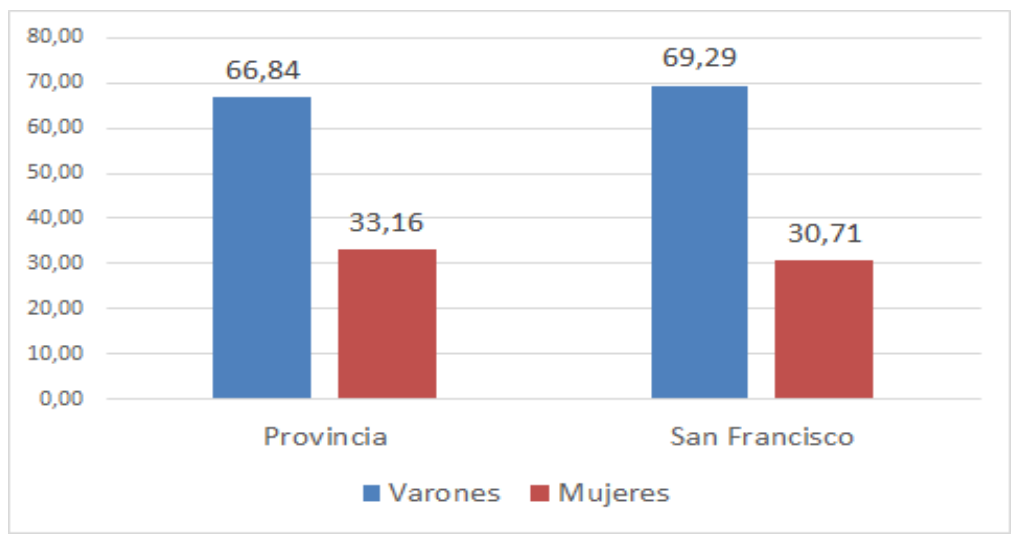

Fuente: elaboración propia en base a Censo 2010.

Alrededor de 690.000 varones eran jefes de hogar en la provincia de Córdoba, mientras que poco más de 340.000 en la misma condición eran mujeres. Una proporción similar se registró en San Francisco. El aumento de la jefatura femenina ha sido indagado en el marco de los cambios en los tipos de hogares. Esto implica un aumento de familias monoparentales femeninas (resultantes de aumentos de divorcios y separaciones de uniones consensuales, situaciones en las que mayoritariamente las mujeres quedan con la tenencia de los hijos).Existe una linea de indagaciones que entienden que los hogares con estas características suelen enfrentar situaciones de vulnerabilidad económica debido a que son "mujeres cabeza de familia" las que deben proveer ingresos y realizar el trabajo doméstico para el grupo familiar (MAZZEO, 2007). Pero, desde otro lugar, se abordan las formas de viabilidad económica y social de estos hogares, por ejemplo, en términos de mayor equidad, cooperación, responsabilidad y cohesión junto con una menor presencia de violencia tanto física como verbal que suelen estar presentes cuando en la pareja, la principal aportante es la mujer (GARCÍA Y DE OLIVEIRA, 2005). ${ }^{9}$

\footnotetext{
9 Un dato interesante y transversal a este punto sería ver cuántos de los hogares con jefatura femenina tienen doble provisión de ingresos. De acuerdo al Censo Nacional 2010, el tipo principal de hogar en el país fue la familia nuclear con el $62 \%$, seguido por los hogares extendidos con el 18\%. Además, se registró un incremento de casi 3\% en los hogares unipersonales (17\%) y una reducción de igual magnitud en los hogares familiares, comparado con 2001. Según la cantidad de personas en el hogar, se incrementaron los que tienen de 1 a 3 personas, registrándose disminuciones en el resto. Los hogares nucleares incompletos con jefatura femenina se mantuvieron estables entre 2001 y 2010, en tanto que se duplicaron los nucleares completos (de pareja sola como así también de pareja e hijos), con jefas mujeres, durante el período intercensal. También se incrementó el porcentaje de hogares extendidos con jefatura femenina en
} 
Mujeres y trabajos en el siglo XXI: una aproximación desde datos secundarios| Gabriela Vergara

El promedio de personas por hogar en San Francisco, fue de 2,9\% y se registró un 1,2\% de hogares con hacinamiento; ambos datos están conectados con el envejecimiento de la población ${ }^{10}$.E1 68,2\% era propietario de la vivienda y el terreno (dentro de los cuales, el 10,4\% no tenía escritura) mientras que el $24,1 \%$ eran inquilinos. Si lo comparamos a nivel provincial y nacional encontramos que, Córdoba se ubicó junto con otras provincias en el rango de 65,1-70 en cuanto al porcentaje de propietarios, mientras que a nivel nacional, el 68\% era propietario pero el 16\% alquilaba. Sólo el 15\% tenía una antigüedad en la vivienda menor a 10 años (si consideramos que una de las actividades económicas con buen desempeño después del 2001 fue la construcción, dicho desempeño no se vio reflejado en un mayor acceso a la tenencia de nuevas propiedades). Este porcentaje es menor a los resultados obtenidos a nivel provincial y a nivel país para el 2010. En Córdoba, el 22\% tenía una antigüedad de hasta 10 años, y en el país ascendía al 24,4\% (DIRECCIÓN GENERAL DE ESTADÍSTICAS Y CENSOS, S/D).

En sintesis, observamos que un tercio de los hogares tienen a una mujer como jefa, al tiempo que la cantidad de personas por hogar se reduce. En cuanto a las viviendas, uno de cada cuatro hogares en la ciudad alquilaba y el acceso a viviendas nuevas alcanzó al 15\% de los hogares.

\subsubsection{Mundo del trabajo}

En este sub-apartado repasamos tres variables para caracterizar de modo global algunos aspectos del mundo del trabajo, a saber, condición de actividad, tasa de ocupación y tipo de ocupación por sector y, distribución de la desocupación.

7 puntos, en el período de 9 años. No disponemos al momento de la presente publicación de acceso a información desagregada para la ciudad.

10 En cuanto al hacinamiento en Córdoba se registró un promedio de 3,2 \%, bastante menor al 2001 , que estaba cerca del 4\%. El promedio nacional de personas por hogar se ubicó en 3,3\%, un valor levemente menor al 3,6\% de 2001 y 1990 y, si bien se redujo la cantidad de personas por hogar, se duplicó el porcentaje de hogares por vivienda, que pasó de $6.6 \%$ en 2001 a $12.2 \%$ en 2010 , indicando así una fuerte demanda habitacional (y la imposibilidad de los hogares de acceder a una vivienda o a créditos hipotecarios) que podría corresponderse con la baja antigüedad de las viviendas. 
Cuando se compara al interior de cada categoría la distribución por sexo, advertimos que la condición de actividad tiene una fuerte presencia femenina en dos de las tres categorias, tal como lo muestra el Gráfico 2:

Gráfico 2. Condición de actividad según género. San Francisco, 2010

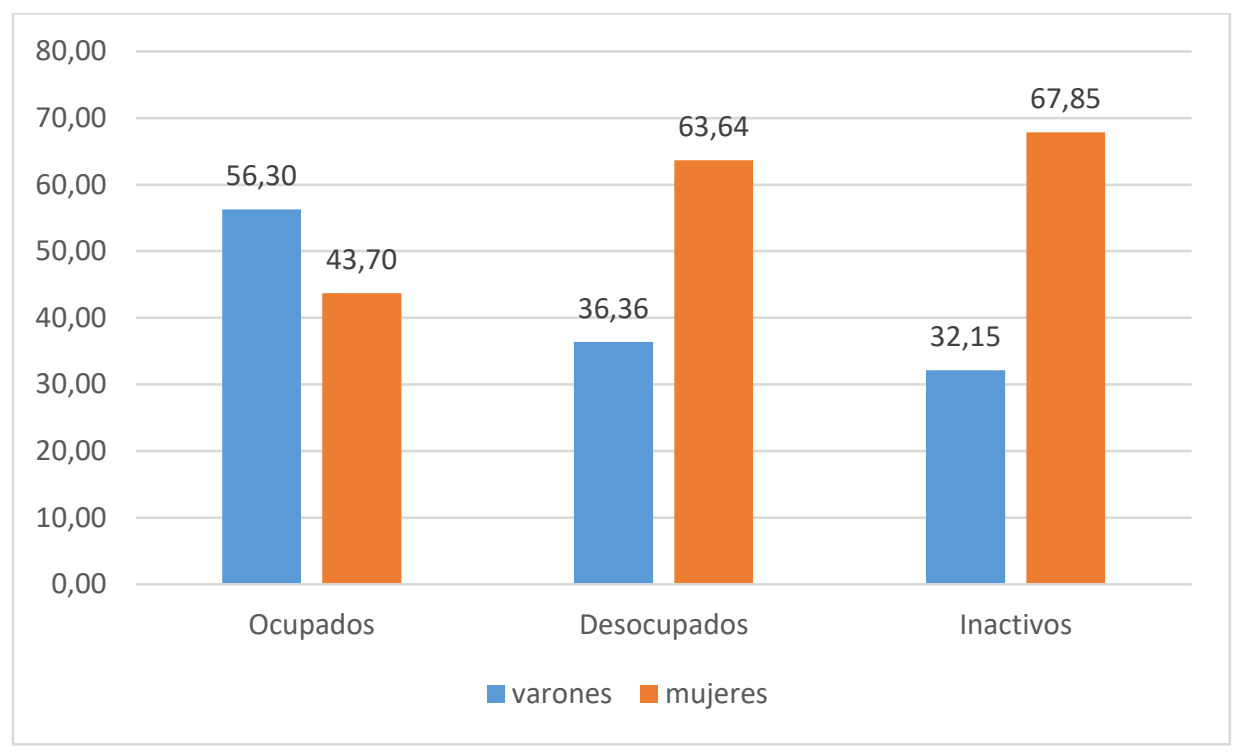

Fuente: Dirección de Estadísticas y Censos de Córdoba (s/d)

Las mujeres casi duplican a los varones tanto en los desocupados como en los inactivos. Dentro de estos últimos, como vimos párrafos arriba hay un porcentaje importante de mujeres adultas mayores con jubilación y cobertura de salud. Ahora bien, si consideramos las tasas de desocupación, también encontramos comportamientos diferenciados. En San Francisco se registró una tasa de $4,52 \%$ como valor general, $2,96 \%$ en varones $y, 6,44 \%$ en mujeres. Cabe recordar que a nivel país se registró una tasa de desocupación de casi seis puntos para el total de la población económicamente activa, que en los varones se ubicó en 4,2\% pero para las mujeres ascendió a 8,2.

En base a relevamientos locales ${ }^{11}$ (INFERENCIAS, 2009) la desocupación en San Francisco registró un descenso de $10 \%$ en 8 años, que se explica principalmente por la reducción de la desocupación masculina, no así la femenina que fue mayor y con porcentajes más elevados en la población de 14

11 Los mencionados relevamientos se efectuaron a pedido de la Municipalidad de San Francisco desde 1997, de manera discontinuada, a una profesional de la ciudad. En 2009, último relevamiento efectuado, los datos resultaron de una muestra aleatoria estratificada de 485 viviendas y 1119 personas. 
a 24 años que en el resto de la población, y particularmente en las mujeres de esas edades.

Gráfico 3.- Desocupación en San Francisco

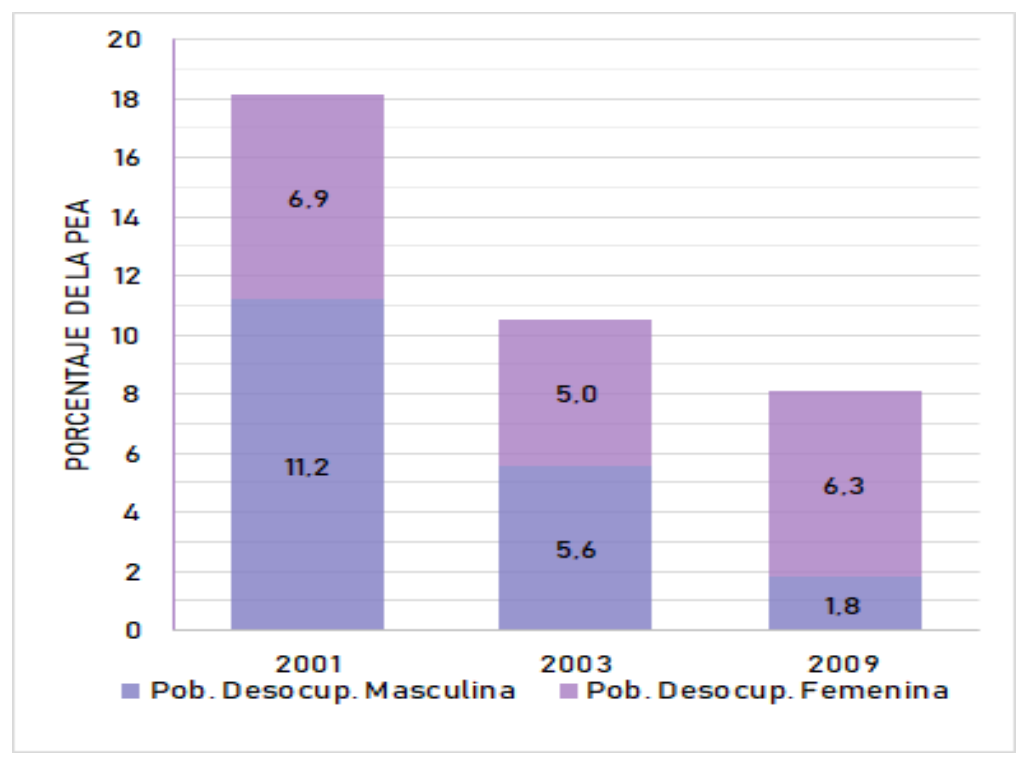

Fuente: Colombo y Peñarrieta (2018).

Las tasas de desocupación descendentes no explican el deterioro del mundo del trabajo, es decir el incremento de la informalidad o empeoramiento de condiciones de quienes sí trabajan. El Censo Provincial de 2008 registró que el $60,9 \%$ de los ocupados san francis que ños manifestaron tener aportes o descuento jubilatorio, cifra mayor al total provincial (50,4\%). Si consideramos la variable edad, entre seis y siete trabajadores de cada diez tenían aportes jubilatorios en el rango etario de 25-59 años. No obstante, la proporción descendía en los más jóvenes, registrándose que en el rango de los 20-24 años uno de cada dos, tenía aportes.

La diferenciación por sexo de la categoría asalariados, da cuenta que, del total de mujeres ocupadas, el $70 \%$ era asalariada, en tanto que en los varones lo era el $63 \%$. La distribución por sector de estos asalariados era la siguiente: 
Mujeres y trabajos en el siglo XXI: una aproximación desde datos secundarios | Gabriela Vergara

Tabla 1. Sector de asalariados según sexo. Censo 2010

\begin{tabular}{l|l|l}
\hline Sector/Sexo & Varones & Mujeres \\
\hline Total & $53,9 \%$ & $46,1 \%$ \\
\hline Público nacional & $54,4 \%$ & $45,6 \%$ \\
\hline Público provincial & $35,2 \%$ & $64,8 \%$ \\
\hline Público municipal & $58,1 \%$ & $41,9 \%$ \\
\hline Privado & $56,3 \%$ & $43,7 \%$ \\
\hline \multicolumn{2}{|c}{ Fuente: Dirección de Estadísticas y Censos de Córdoba (s/d) }
\end{tabular}

La mayor participación femenina en relación de dependencia con el Estado provincial (que abarca el ámbito de salud, justicia, educación, seguridad, entre los principales ámbitos) a nivel ciudad, puede explicarse teniendo en cuenta que la docencia continúa siendo una ocupación prácticamente feminizada. Esto, a su vez, puede corresponderse con el porcentaje elevado de mujeres con nivel educativo terciario completo. Las segregaciones horizontales en el mundo del trabajo se complementan con la búsqueda de cierta estabilidad ${ }^{12}$.

Las dinámicas entre ocupación y desocupación feminizada dan cuenta de metamorfosis que se inscriben en una mayor informalidad y precarización del trabajo y, en cierta segmentación intragénero. Estas transformaciones en el mundo del trabajo que afectan a jóvenes mujeres, con desempleo o empleo no registrado impactarán en el sistema previsional (que se asemeja a la pirámide de población), como así también, en la composición de los hogares/viviendas.

\section{Consideraciones finales}

En este artículo nos propusimos conectar datos secundarios de una ciudad del interior de Argentina para conformar un escenario de procesos estructurales que operan en los cruces del mundo del trabajo y el mundo reproductivo, en el que se inscriben las prácticas cotidianas de trabajos de

12 Así se interpreta una tendencia parecida en Brasil, donde dentro del segmento nivel educativo universitario, las mujeres superan en 8 puntos a los varones en la categoría de funcionarios públicos, mientras que, en el cuentapropismo, los varones superan en casi 7 puntos a las mujeres. Esta concentración diversa remite a estrategias diferentes, puesto que el empleo estatal otorga previsibilidad, seguridad y menores constreñimientos en las situaciones del trabajo reproductivo, por ejemplo, en el caso de la maternidad (Guedes, 2015). Una mirada similar, pero en una ocupación distinta, es el de las mujeres que prefieren los trabajos por cuenta propia que pueden realizar en sus hogares, donde no cuentan con estabilidad laboral pero si con la posibilidad de articular demandas de ambos trabajos. Esto abarca desde actividades precarias y de baja calificación como la costura para empresas de ropa, el armado de broches, hasta el pequeño almacén, kiosco o tienda de ropa que se instala en una parte de la vivienda. 
varones y mujeres, del siglo XXI. Tal como lo hemos explicitado párrafos arriba, esta descripción pretende articular las particularidades y generalidades, en tanto ciudad neocolonial.

Si bien existen limitaciones en cuanto a la disponibilidad de datos y su actualización a nivel ciudad (tales como tipos de hogares, tipo de contratación en el sector privado y distribución por género en distintas actividades económicas, formas de contratación en el sector público, entre otros) la información expuesta páginas arriba nos permite problematizar las relaciones entre tres nodos: el mundo del trabajo (donde se concretiza el trabajo productivo organizado por relaciones de capital/trabajo), los hogares y las viviendas (donde reside principalmente la fuerza de trabajo pasada, presente y futura, y donde se realiza una parte del trabajo reproductivo) y la población en sus características más básicas, pero no por ello, menos relevantes.

Lo antes expuesto nos permite describir tres procesos convergentes: a) el contraste en términos demográficos y de formalidad entre un grupo etario de adultos mayores (principalmente mujeres, con cobertura previsional y de salud) por un lado, y jóvenes adultos con ocupaciones informales, incluido el cuentapropismo ( $\sin$ aportes ni cobertura de salud). Ante esto nos preguntamos por los circuitos de cuidado para mujeres adultas mayores y, por la posibilidad de que estos hogares (con coberturas e ingresos estables) contribuyan a amortiguar el deterioro de las condiciones de las siguientes generaciones; b)la presencia de empleo público provincial femenino y la feminización de la docencia como uno de los lugares donde en los que todavía persisten estabilidad laboral y empleo formal; c) hogares sin vivienda propia, con jefatura de hogar femenina en aumento y menor cantidad de hijos. Los hogares nucleares completos deben desplegar estrategias de generación de ingresos con dos proveedores de manera inevitable e indiscutible, tanto más porque casi cuatro de cada diez no tenían casa propia. Y la tendencia a una menor cantidad de hijos por hogar se dirime entre los tiempos para proyectos personales, lo complejo de la doble presencia y la calidad de la crianza. Los hogares incompletos con jefatura femenina claramente enfrentan dilemas múltiples 
desde ingresos, tiempos y distribución de tareas. La interrelación de estos fenómenos podría indicar un paulatino deterioro del empleo estable y la persistencia de formas diversas de ocupaciones con menores niveles de previsibilidad y estabilidad que dan cuenta de una desarticulación de la sociedad salarial con una fuerte presencia de las mujeres en el mundo del trabajo y en los hogares.

Estos ejes operan a modo de interrogantes/hipótesis que podrán ser profundizados con datos estadísticos actualizados y con una lectura en términos de clases sociales, que permita comprender las maneras particulares en que se configura la desigualdad en el presente siglo.

\section{Referencias:}

ALCAÑIZ MOSCARDÓ, Mercedes."Trayectorias laborales de las mujeres españolas. Discontinuidad, precariedad y desigualdad de género". La Ventana, núm.46, pp.244-285, 2017.

ANTUNES, Ricardo. Los sentidos del trabajo. Buenos Aires: Herramienta, 2005.

ASE, Iván. La Descentralización de Servicios de Salud en Córdoba (Argentina): Entre la Confianza Democrática y el Desencanto Neoliberal. Salud colectiva, 2(2), 199-218, 2006.

BINSTOCK, Georgina. "Avatares de las familias argentinas: evidencias a partir del Censo 2010”, en Población, marzo, pp.25-33, 2013.

BOERI, Natascia. Challenging the gendered entrepreneurial subject: Gender, Development and the Informal Economy in India. Gender \& Society, vol.XX, nro.10, 2018.

CARRASCO, Cristina. La sostenibilidad de la vida humana: ¿un asunto de mujeres?. IN Magdalena León.Mujeres y trabajo: cambios impostergables. Porto Alegre:Veraz comunicaçao, 2003.

CENSO PROVINCIAL DE POBLACIÓN 2008. Series resultados a nivel municipal y comunal. Municipio de San Francisco. Dirección General de Estadísticas y Censos. Disponible en: https://www.sanfranciscodigital.com.ar/documentos/censo-provincial-2008san-francisco.pdf

CEPAL. Proyecciones de población. Observatorio Demográfico. 2014. Disponible en: 
https://repositorio.cepal.org/bitstream/handle/11362/39228/1/S1500643_m $\underline{\text { u.pdf }}$

COLOMBO, Andreínay PEÑARRIETA Jimena. "Feminización del trabajo en San Francisco (2001-2017): una mirada desde la Sociologia de los cuerpos/emociones"en Ana Lucía Cervio y Victoria D’hers (comps.) Sensibilidades y experiencias: acentos, miradas y recorridos desde los estudios sociales de los cuerpos/emociones. CABA: ESE Editora. pp121-144, 2018.

DE SENA, Angélica."Las mujeres ¿protagonistas de los programas sociales? Breves aportes a la discusión sobre la feminización de las politicas sociales", en Angélica De Sena (edit) Las politicas hecha cuerpo y lo social devenido emoción: lecturas sociológicas de las políticas sociales. Buenos Aires: ESE Editora. pp.99-126, 2014.

DIRECCIÓN DE ESTADÍSTICAS Y CENSOS DE CÓRDOBA (s/d) "Censo Nacional 2010. Sintesis de Cabecera. San Francisco". Disponible en: https://datosestadistica.cba.gov.ar/dataset/eaab30e4-d400-465d-bf53e9094558f3d2/resource/515c872f-ab6a-480f-8d23c39a7ba20186/download/desd_cn2010_san_justo_-san-francisco_cabecera.pdf

FRAIRE, Vanina, MAGNANO, Cecilia, COLOMBO, Andreína y PEÑARRIETA, Jimena. Datos censales y aglomerado. Un análisis de datos secundarios para comprender el mundo del trabajo. IN: Gandía, C. et al.Metodologias de la investigación: Estrategias de indagación II. Buenos Aires: ESE Editora, 2018.

GARCÍA, Brígida y DE OLIVEIRA, Orlandina. Mujeres jefas de hogar y su dinámica familiar. Papeles de Población, 11 (43), 29-51, 2005.

GARCÍA VILLANUEVA, Jorge; ÁVILA RODRÍGUEZ, Diocelina; VARGAS PÉREZ, Martha Karina; HERNÁNDEZ RAMÍREZ, Claudia Ivonne. "Acerca de la feminización de profesiones. Caso: la docencia en preescolar en la Ciudad de México”. La ventana, vol. V, núm. 42, julio-diciembre, pp.129-151, 2015.

GUEDES, Moema de Castro "Heterogeneização da mão-de-obra feminina: o que mudou no período recente?" Revista Género, vol.15, núm2. Pp139-154, 2015.

GUEDES, Moema de Castro. Perceptions of the State's Role, Productive and Reproductive Work: An Analysis of Rio de Janeiro. Cadernos Pagu, (47), september 15, 2016.

INFERENCIAS. Análisis del Mercado Laboral de San Francisco. San Francisco: Municipalidad de San Francisco. Mimeo. 2009. 
Mujeres y trabajos en el siglo XXI: una aproximación desde datos secundarios | Gabriela Vergara

HIRATA, Helena. "Emprego, responsabilidades familiares e obstáculos sócioculturais à igualdade de gênero na economia". Revista do Observatório Brasil da Igualdade de Gênero, Brasília, SPM, vol.1, 2010.

MAZZEO, Victoria. "Los cambios en la organización familiar: el incremento de las familias monoparentales en la Ciudad de buenos Aires a partir de los ochenta”. Población de Buenos Aires, 4 (5), 63-74, 2007.

MINISTERIO DE SALUD DE LA PROVINCIA DE CÓRDOBA. (2011) Manual de Inducción. Disponible en: http://multimedia.cba.gov.ar/web/MANUAL\%20DE\%20INDUCCION\%20SALU $\underline{\text { D.pdf }}$

MONTOYA, Óscar Luis "El envejecimiento de la población y sus efectos en los requerimientos de equipamiento urbano de educación básica en Aguascalientes, México", en Estudios demográficos y urbanos, vol. 27, núm. 1, enero-abril, pp. 119-152, 2012.

PAZ, Jorge A. "Los desafios laborales del envejecimiento de la población en América Latina y el Caribe”, en Revista Latinoamericana de Población, vol. 5, núm. 9, julio-diciembre, pp. 123-144, 2011.

PICCHIO , A. El trabajo de reproducción, tema central en el análisis del mercado de trabajo. En C. Borderias, C. Carrasco, \& C. Alemany, Las mujeres y el trabajo: rupturas conceptuales Madrid: Fuhem-Icaria. pp. 453-490, 1994.

VERGARA, Gabriela. "Experiencias de la doble jornada en mujeres recuperadoras de residuos de Córdoba en la actualidad. Un análisis de sus tramas corporales, percepciones y emociones". Tesis de Doctorado. Facultad de Ciencias Sociales. Universidad de Buenos Aires. Inédito, 2012.

VERGARA, Gabriela. "Ahora hay trabajo ... pero no es muy remunerativo". Hacia un puzzle de datos acerca de los procesos de estructuración como vía para pensar la hermenéutica en la Sociologia”. En Gandía, Claudia, Vergara, Gabriela, Lisdero, Pedro y Quattrini, Diego (comps.) Metodología de la investigación: estrategias de indagación II. Buenos Aires: ESE Editora. Pp.111133, 2018a.

VERGARA, Gabriela "Trabalhadoras na cidade: interpretações sobre tempos, trajetórias e percepções "no" século XXI", Ciencias da Sociedade. Vol.2, núm.3, 2018b.

VERGARA, G;COLOMBO, A; MANAVELLA, A. y PEÑARRIETA, J. "Configuraciones de sensibilidades en trabajadoras del siglo XXI: aproximaciones desde ciudades del interior de Argentina". Ponencia presentada en el Encuentro "Ciencias Sociales, Sensibilidades y Sociedades", organizado por el CIES, realizado en Toronto (Canadá), los días 13 y 14 de julio de 2018. Inédito. 
Mujeres y trabajos en el siglo XXI: una aproximación desde datos secundarios| Gabriela Vergara

WAINERMAN, Catalina y NAVARRO, Marysa. El trabajo de la mujer en la Argentina: un análisis preliminar de las ideas dominantes en las primeras décadas del siglo XX. Buenos Aires: CENEP, 1979. 